\title{
Nebulae (emission / absorption)
}

\section{Courtney Wrenn}

\begin{abstract}
My presentation consists of a collage on panel. Using colour laser copies of nebulae, the dual composition recombines torn pieces of several nebular images. Optically entangled from light colour patterns, the focus of each nebula resembles the opposing behaviour of absorption and emission nebula. The collages include false colour composite astronomical images to extend the visualization beyond the limits of the human eye. The subtleties of infrared and $\mathrm{x}$-ray interpreted light appear to observe extra-dimensions that we intellectually understand. The layered torn paper creates forms for visual experience/contemplation. The pseudonym, scrapworm, names the allegory of my creative process. This identity is a human perspective on witnessing the passage of time and figuratively identifies with more subtle entanglements of living systems dynamics. I have recently been working with astronomical data acquired by space telescopes in my fine art collage work. The nebulae collages seek to create discourse on the time-bending implied by transmitting optical data through deep space (as related to the limit the of speed of light as well as emerging paradigms on the electric universe, quantum dynamics, and null physics).
\end{abstract}

My interdisciplinary projects are motivated by explorations in astronomical order (including scientifically observable structures/motions, as well as more abstract suggestions such as David Bohm's Implicate Order in the universe) combined with reference to the progressed history of optical technologies and photographic image capture. Photographic imaging has become engrained in human cultural cognitions almost as pervasively as the orders of the cosmos are embedded in the bio-geologic evolutions of life on earth.

As a lived process, my creative work is ultimately a discourse on time and human experience. Nebulae (4' x 8', below) acted as catalyst for my 2009-10 series of handmade, large-scale, colour montages, and was also primarily created for INSAP VII display.

Courtney Wrenn, 'Nebulae (emission / absorption)', eds. Nicholas Campion and Rolf Sinclair, Culture and Cosmos, Vol. 16 nos. 1 and 2, 2012, pp. 439-442. www.CultureAndCosmos.org 


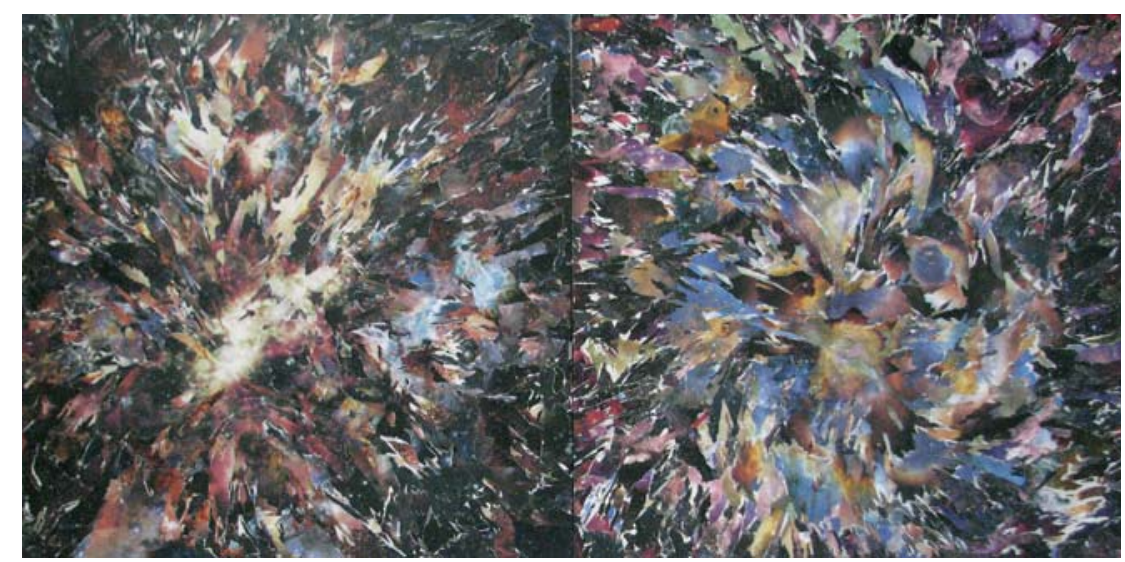

Figure 1. Nebulae, 2010, 48” x 96” acrylic medium and paper on panel

Use of nebular imagery in my physical montage work developed within my employment as a live star-show planetarium instructor (2008-2011). At the Andrus Planetarium (Hudson River Museum, Yonkers, NY), I have had freedom to develop original content for presentation with the $\mathrm{AV}$ systems and Zeiss star projector. My focus as an educator has evolved with my experience in the position to encourage, for attendees of all ages, realizations of scale-shifts and simultaneous perspectives. My reference to multiple perspectives includes use of an orrery and animated media to discuss the differences between earthbound and 'bird's eye' views, also comparing bird's eye views of the Solar System and the Milky Way to our earthbound view of their contributing parts (neighboring planets and local stars, respectively) for comprehensive understanding.

Alternating between scales and views, my seasonally changing presentations of deep sky objects informed my conceptual framework for the Nebulae montage piece. This framework-'emission :: absorption'intends to investigate how we relate to visual media by detecting subtle contrasts and patterns, also questioning how we understand the known and unknown worlds, as enhanced by mechanical devices. Similar dualised themes therefore include 'internal and external awarenesses'. In subsequent works I expand on the metaphor of absorption nebulae (such as the Horsehead or Keyhole Nebula) that are visible only when lit from behind (from an earthbound, or 'earth orbit area' perspective). Ongoing works in the series proceed to represent multiple dimensions simultaneously. 
These literal and figurative 'layerings' collapse in a décollage sense of decay while, also expanding toward the viewer with optical motions and visual symbolisms. The study of astronomy is a glimpse of the past coming into the present (both as light-years and in accumulated scholarship); these works exist in similarly blended theoretical timescales by combining frames (or moments) captured by the movement of light, over time.

These photographic stills are frozen in time as 'past,' but also look towards the 'future' as my interactive processes and projects unfold. I shoot hundreds of images as a 'daily practice' that explores digital proliferation, 'reality' framing and latent (filmic) imaging processes. Almost like interpreting the numbers being received from a space telescope's processors, I map visual images via intuitive pattern-forming that seeks to represent mental and tangible motion through observational 'meta-lenses'. My process involves printing on paper, making color laser blowups and building up torn and cut surfaces.

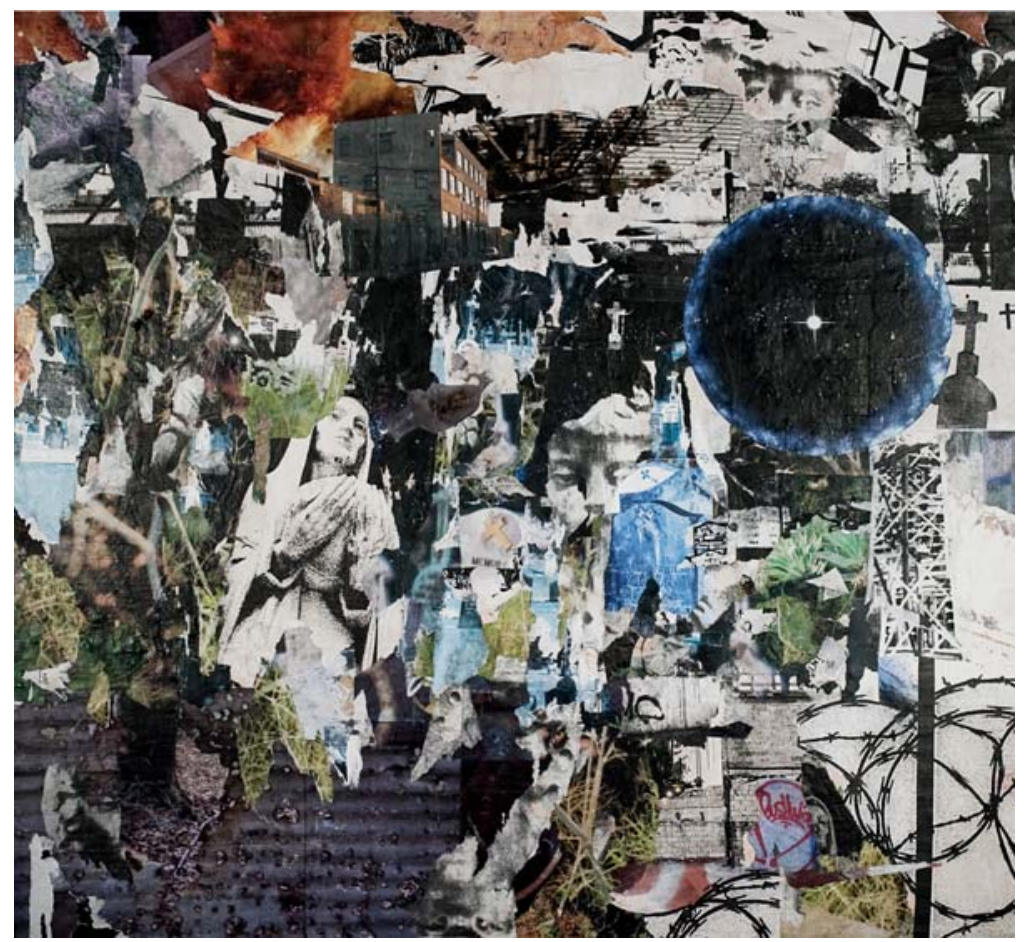

Figure 2. Trinity industry, 2010, 30”x28” acrylic medium and paper on panel 
442 Nebulae (emission / absorption)

Responding to McLuhan's 'medium as message,' my imagery and décollage methods evoke decay, interconnectedness, memory and perpetual blending of the building blocks of matter (alluding to atomic elements, cosmic dust and kinetic energy).

The compositions are intended to create optical and associative spatial dimensions that appear to come forward and recede, though on one plane or 'brane' (as in 'braneworld' quantum theories, such as physicist Lisa Randall's theses). Having worked with the allegorical pseudonym scrapworm for nine years, my most recent bodies of work are being shown under a new pseudonym sean: ambiguous and with wrenn, the name is similar to the spoken sound of scrapworm. My previous bodies of work focused on media ecology as psychological, historical progressions of national identity (based on my 2003-2005 displeasures with global American attitudes). Throughout my MFA thesis work, my inquiry progressed from the sociological meta-language of media into increasingly metaphysical discourses on interconnected living systems and the evolution of consciousness.

www.scrapworm.info 